\title{
Exercise capacity in apparently healthy survivors of cancer
}

\author{
E De Caro, F Fioredda, M G Calevo, A Smeraldi, M Saitta, G Hanau, M Faraci, \\ F Grisolia, G Dini, G Pongiglione, R Haupt
}

See end of article for authors' affiliations

\section{Correspondence to:} Dr G Pongiglione, Dept of Cardiology, "G. Gaslini" Children's Hospital, Largo Gerolamo Gaslini 5, 16148 Genoa, Italy; giacomopongiglione@ ospedale-gaslini.ge.it

Accepted

12 September 2005

Published Online First

27 September 2005

\begin{abstract}
Aims: To evaluate cardiopulmonary exercise tolerance in a large cohort of apparently healthy paediatric cancer survivors in order to determine their participation in sporting activities.

Methods: A total of 84 young ( $<21$ years) asymptomatic childhood cancer survivors, who had been exposed to anthracyclines (mean dose $212 \mathrm{mg} / \mathrm{m}^{2}$ ) and/or chest irradiation (median dose $2000 \mathrm{cGy}$ ), with normal left ventricular systolic function at rest (fractional shortening $>29 \%$ ), and 79 healthy controls were studied. Exercise testing was performed on a treadmill ergometer. Gas exchange analysis and derived variables were measured on a breath-by-breath basis. Pulmonary functional evaluation was performed before exercise. Echocardiographic evaluation at rest was performed within one month before the exercise test.

Results: There were no differences in exercise responses between patients and controls. In boys <13 years, mean $\mathrm{VO}_{2}$ max was slightly but significantly lower than in controls. This finding was thought to be a result of decreased physical fitness as all the other exercise parameters were similar to those in the controls.

Conclusions: Results show that apparently healthy survivors of paediatric cancer can take part in dynamic sporting activities if they exhibit a normal response to cardiopulmonary exercise testing, while those that exhibit a reduced $\mathrm{VO}_{2}$ max should be re-evaluated after an aerobic training programme, and should undergo tailored dynamic physical activity if the $\mathrm{VO}_{2}$ max does not normalise.
\end{abstract}

$\mathrm{P}$ hysical activity is important for a healthy lifestyle in children, particularly in survivors of cancer, who need to regain psychophysical wellbeing. ${ }^{1-3}$ However, exercise capacity can be reduced in these patients. ${ }^{4-7}$ Many have received chemotherapy with anthracycline containing regimens and/or chest irradiation and are thereby exposed to the attendant risk of pulmonary dysfunction and late onset cardiac toxicity. ${ }^{89}$ Although the risk is mainly related to the cumulative dosage received, no safe exposure limit exists, and the lack of symptoms and a normal cardiac systolic function at rest cannot guarantee the absence of any cardiac impairment. ${ }^{10-12}$ Exercise can unmask subclinical myocardial dysfunction, which may be adversely affected by an inappropriate physical activity. ${ }^{13}{ }^{14}$ However, other factors, such as musculoskeletal dysfunction, lack of physical fitness, and psychological attitude can limit the exercise capacity of these patients, which, in many instances, can be positively affected by physical activity. ${ }^{15}$ A thorough functional evaluation is thus warranted to determine an appropriate level of physical activity for these patients. The aim of this study was to evaluate the cardiopulmonary exercise tolerance in a cohort of children and young adult survivors of paediatric cancer who had received cardiotoxic drugs, and were asymptomatic with normal systolic cardiac function at rest, in order to obtain information on their participation in sporting activities.

\section{METHODS}

Subjects

Survivors of childhood cancer who had been treated at the Istituto Giannina Gaslini Children's Hospital in Genova between 1976 and 2000 and were assessed in the off-therapy department between 2000 and 2002 were studied. Inclusion criteria were: to have received chemotherapy with anthracyclines and/or radiotherapy to the chest as part of the treatment, which had to be discontinued at least one year before assessment; to be in complete continuous remission; to be asymptomatic; and to have a normal echocardiographic evaluation at rest. A total of 178 patients fulfilled the study requirements. Subjects aged 7-21 years and who were physically able to perform the exercise capacity test were eligible for the study. The control group consisted of healthy, non-athletic but physically active subjects with innocent murmurs who were examined for participation in sporting activities and had a normal echocardiographic examination at rest; they were comparable for sex and age.

For each eligible patient, information was collected on primary diagnosis, cumulative dose of anthracyclines received, and dose and field of chest irradiation. Since the anthracycline derivatives used have different cardiotoxic effects, the cumulative dose $\left(\mathrm{mg} / \mathrm{m}^{2}\right)$ was calculated as Adriamycin (ADR) equivalent. ${ }^{16}$ For cases and controls information was also collected on gender, age, weight and height at the time of the test, and body mass index (BMI) calculated as centiles. ${ }^{17}$ The test was performed after explanation of the exercise protocol to the subjects and their families; informed consent was obtained from all subjects.

\section{Echocardiography}

Echocardiographic evaluation at rest was performed within one month before the exercise test, utilising a Philips Sonos 5500 ultrasound system. Left ventricle dimensions were determined in the short axis of parasternal view and left ventricular systolic function was expressed as fractional shortening (FS), which was considered normal if $\geqslant 29 \%{ }^{18}$

\section{Exercise test}

The exercise test protocol was performed in the morning, at least two hours after the last meal. To determine the relative physical fitness of each subject, information was collected on 


\begin{tabular}{|c|c|c|}
\hline & $\begin{array}{l}\text { Survivors } \\
(\mathrm{n}=84)\end{array}$ & $\begin{array}{l}\text { Controls } \\
(\mathrm{n}=79)\end{array}$ \\
\hline \multicolumn{3}{|l|}{ Gender, n ((\%) } \\
\hline Male & $58(69)$ & $53(67)$ \\
\hline Female & $26(31)$ & $26(33)$ \\
\hline \multicolumn{3}{|c|}{ Age group at test, mean (SD) } \\
\hline $7-13 y$ & $10.2(1.4)$ & $10.1(1.6)$ \\
\hline $14-21$ y & $16.5(2.2)$ & $16.2(2.5)$ \\
\hline \multicolumn{3}{|c|}{ BMI $>95$ th centile, $n(\%)$} \\
\hline Males, $7-13$ y & $11(52)$ & $9(29)$ \\
\hline Males, $14-21$ y & $11(30)$ & $4(18)$ \\
\hline Females, $7-13 y$ & $2(17)$ & 3 (19) \\
\hline Females, $14-21$ y & $2(14)$ & $3(30)$ \\
\hline
\end{tabular}

the patient's level of physical activity. Pulmonary functional evaluation, including spirometry and single breath carbon monoxide diffusing capacity, was performed before exercise. Exercise testing was then performed on a Galaxy MTC Climb 2000 treadmill ergometer, using a modified Bruce protocol. At the first stage the treadmill speed and slope were $1.7 \mathrm{mph}$ and $0 \%$, respectively, to allow the patients to warm up. At the second stage the treadmill slope was increased to $10 \%$; thereafter slope and speed were increased according to the Bruce protocol. ${ }^{19}$ Blood pressure was measured utilising a mercury sphygmomanometer with an appropriately sized cuff at rest, at the end of each stage during exercise and until return to baseline into recovery. Heart rate and rhythm were monitored continuously throughout exercise and until return to baseline into recovery utilising a Cardiosoft Hellige Marquette 12 exercise electrocardiographic system. Oxygen consumption $\left(\mathrm{VO}_{2}\right)$, carbon dioxide production, minute ventilation (VE), and derived variables were measured on a breath-by-breath basis utilising a Sensormedics Vmax 229 cardiopulmonary exercise testing system, which was calibrated before each test. Every patient was strongly encouraged to continue to the point of exhaustion; a respiratory exchange ratio $(\mathrm{RER}) \geqslant 1.1$ was regarded as an indicator of maximal aerobic performance. The $\mathrm{VO}_{2}$ at the peak of the exercise was defined as maximal $\mathrm{VO}_{2}\left(\mathrm{VO}_{2} \max \right)$. The anaerobic threshold (AT) was determined using the ventilatory equivalent method or the $\mathrm{V}$-slope method, depending on which was better displayed on the diagram.

\section{Statistical analysis}

Data are presented as mean (SD) or median and range for continuous variables, and as absolute and relative frequencies for categorical variables. The ANOVA $t$ test for continuous variables, and the $\chi^{2}$ or Fisher exact test for categorical variables were used to measure differences between cancer survivors and controls. A p value $<0.05$ was considered significant based on two-tailed tests. The SPSS statistical software program was used for all analyses.

\section{RESULTS}

Of 148 eligible patients, 84, all living within $200 \mathrm{~km}$ of our institution, agreed to participate; they formed the study group. For comparison, 79 healthy controls studied in the last three months were included in the study. The two groups were comparable for sex, age, and BMI (table 1).

Over half of the male survivors $<13$ years of age at the exercise test had a BMI $>95$ th centile compared to only $29 \%$ of the controls. The primary diagnosis of cancer survivors was acute lymphoblastic leukaemia $(\mathrm{n}=34)$, Hodgkin's disease $(n=17)$, non-Hodgkin's lymphoma $(n=9)$, acute nonlymphoblastic leukaemia $(n=4)$, Ewing's sarcoma $(n=7)$,
Wilms' tumour $(n=4)$, neuroblastoma $(n=3)$, rhabdomyosarcoma $(\mathrm{n}=2)$, hepatoblastoma $(\mathrm{n}=2)$, osteosarcoma $(\mathrm{n}=1)$, and myelodysplasia $(\mathrm{n}=1)$. Their mean age at diagnosis was 6.4 (4.1) years (range 25 days to 16.8 years), and their mean interval since the completion of anticancer treatment was 6.4 (3.4) years (range 1.0-15.3 years). Among those treated with anthracyclines, the mean cumulative dosage was $212(100) \mathrm{mg} / \mathrm{m}^{2}$ (range 64-545 $\mathrm{mg} / \mathrm{m}^{2}$ ); in those treated with chest radiotherapy, the median dose received was 2000 cGy (range 1000-4300 cGy, mean 1840 (775.6)). Table 2 shows the distribution of subjects according to potentially cardiotoxic treatments received and mean doses received of anthracyclines within each category.

The echocardiographic parameters of survivors at rest were within normal range with a mean FS of $36.5 \%( \pm 3.7)$. Pulmonary function at rest was normal in all except two survivors, who had a very mild restrictive pattern $(86 \%$ and $88 \%$ respectively of the predicted total lung capacity). All survivors and controls were engaged in some physical activity, with younger children in either group mostly involved in leisure activities. Table 3 presents exercise results.

All subjects exercised to the point of exhaustion. None developed cardiac symptoms or showed significant arrhythmias other than infrequent and isolated premature atrial or ventricular contractions, normally during the first stages of exercise or into recovery. There were no differences in exercise test results between patients and controls. Survivors $<13$ years of age had a mean value of $\mathrm{VO}_{2}$ max significantly lower than controls of the same age (34.0 (5.7) $v$ 38.9 (6.7); $\mathrm{p}<0.001)$. This difference was present only in male subjects (33.9 (6.0) v 40.8 (6.1); p < 0.0001), and disappeared among females (34.1 (5.5) v 35.3 (6.6); NS).

Only eight survivors ( six males and two females), had $\mathrm{VO}_{2}$ $\max$ values $<5$ th centile of the controls. Their cumulative dose of anthracyclines was $189-330 \mathrm{mg} / \mathrm{m}^{2}$, and only one patient had received chest radiotherapy at a cumulative dose of 2000 cGy in association with $210 \mathrm{mg}$ of anthracyclines. In all the BMI was $>95$ th centile for sex and age.

\section{DISCUSSION}

Regular participation in physical activity is one of the strategies recently recommended by the American Heart Association for promoting cardiovascular health in childhood. Activities that generate energy expenditure $\geqslant 50-60 \%$ of maximal exertion should be encouraged, and children with physical limitations should be included in activities specifically tailored to their physical ability. ${ }^{1}$ Survivors of paediatric cancer need to recover complete psychophysical growth, and therefore should be directed towards physical activity. However, late cardiotoxicity is a very important issue in such patients.

Differences exist among individuals in their sensitivity to the anthracycline damage, and even if cardiac changes are more likely to be observed after a cumulative dose $>350-400$ $\mathrm{mg} / \mathrm{m}^{2}$, no safe dose exists since cardiac toxicity can be present with doses $<180 \mathrm{mg} / \mathrm{m}^{2} .^{20-22}$ Moreover, subclinical cardiac damage can be present despite the lack of symptoms and with a normal cardiac function at rest, and it is conceivable that regular physical activity of moderate or high intensity can negatively affect the natural history of the cardiac damage. Recommendations regarding participation in sporting activities are therefore very difficult in apparently healthy childhood cancer survivors who have received low dose anticancer treatment, as that an unproven restriction of their physical activity may be perceived by these patients as a decrease in their quality of life. ${ }^{23} 24$

Subclinical myocardial dysfunction can be unmasked under stress. Abnormalities of ventricular function, not detected or less pronounced at rest, have been shown in 
Table 2 Distribution of survivors by cardiotoxic treatment

\begin{tabular}{|c|c|c|c|c|c|}
\hline \multirow{2}{*}{$\begin{array}{l}\text { ADR }\left(\mathrm{mg} / \mathrm{m}^{2}\right) \\
\text { equivalent }\end{array}$} & \multicolumn{4}{|c|}{ Radiotherapy to the cardiac area (cGy) } & \multirow{2}{*}{$\begin{array}{l}\text { ADR equivalent mean } \\
\text { cumulative dose }\left(\mathrm{mg} / \mathrm{m}^{2}\right)\end{array}$} \\
\hline & None & $<\mathbf{3 0 0 0}$ & $3000-5000$ & Total & \\
\hline None & - & 1 & - & 1 & - \\
\hline$<180$ & 14 & 13 & 2 & 29 & $130(28.7)$ \\
\hline $180-360$ & 36 & 10 & 1 & 47 & $231(49.4)$ \\
\hline$>360$ & 2 & 5 & - & 7 & $458(56.6)$ \\
\hline Total & 52 & 29 & 3 & 84 & $212(100)$ \\
\hline
\end{tabular}

anthracycline treated patients by both exercise echocardiography and dobutamine stress echocardiography, in terms of a reduced increase of the fractional shortening and of a reduced end systolic left ventricular posterior wall dimension and percentage thickening, respectively. ${ }^{25-30}$ The usefulness of these techniques is limited to the evaluation of cardiac function and their clinical significance has yet to be ascertained. The role of the exercise test is still controversial because of the poor correlation of ventricular function with exercise capacity. Patients with heart failure can have a normal or near normal exercise capacity depending on several factors producing positive changes at the muscular, vascular, and neurohormonal level. ${ }^{31}{ }^{32}$ Thus, even if there is limited data available from patients with anthracycline associated cardiomyopathy, exercise performance may be an unreliable surrogate for cardiac function in this population. ${ }^{33}$ However, cardiopulmonary exercise testing (CPET) addresses the body's global physiological adaptation to exercise, and it can therefore unmask compensatory mechanisms for the abnormal cardiac function. ${ }^{34} \mathrm{VO}_{2}$ max is the best available index of functional exercise capacity, and even though in early mild cardiovascular disease the peak $\mathrm{VO}_{2}$ may still be normal, other specific physiological variables, such as AT and $\mathrm{O}_{2}$ pulse may become abnormal. ${ }^{34}{ }^{35}$ Hauser et al performed exercise echocardiography and spiroergometry on 38 patients, and found a good correlation between cardiac function and exercise performance. In their study, exercise capacity, as measured by $\mathrm{VO}_{2}$ max, was similar to that of controls in the 28 patients that had a normal stress echo, but was significantly lower in the 10 patients who had decreased FS and ejection fraction after exercise. ${ }^{36}$

However, exercise capacity can be reduced in a large percentage of these patients by several factors other than cardiac toxicity, such as respiratory or musculoskeletal dysfunction, psychological attitude, lack of physical fitness, endocrine deficiencies, chronic fatigue, or peripheral neuropathies. Most of these not only do not contraindicate but can even be positively affected by a regular physical activity, even of moderate intensity. ${ }^{2}{ }^{37-42}$ Moreover, early mild heart disease can be difficult to distinguish from lack of fitness, and response to an aerobic training regimen may help to distinguish between these two clinical entities. ${ }^{43}{ }^{44}$ Finally, patients with heart failure can improve their exercise capacity with a tailored exercise training programme. ${ }^{32}$ In case of exercise intolerance, integrative CPET has the capability to address many questions about the limiting mechanism(s), and provides information not only of the patient's anaerobic threshold, but also of the level of exercise that the patient can perform without undue stress. Thus, the test results can guide the physician in both diagnosis and exercise prescription; we therefore believe that CPET may be the most useful initial test for the functional evaluation of these patients.

In our study, which is to our knowledge one of the largest in which CPET was carried out in paediatric cancer survivors, none in the group of older patients showed an abnormal exercise response, possibly because of an insufficient length of follow up. It is known that the incidence of cardiac abnormalities increases with time, and more than half of the patients exposed to anthracyclines will show cardiac abnormalities at 10-20 years from diagnosis; in this subgroup of our patients the mean interval since diagnosis was only 7.7 (3.4) years. ${ }^{22} 4546$ Another possible explanation could be the low mean cumulative dose of anthracyclines that the patients had received, although any dose of anthracyclines can be cardiotoxic. Johnson et al assessed the long term impact of paediatric low dose anthracycline therapy on the circulatory response to moderate exercise, and found that the $\mathrm{VO}_{2}$ max was significantly lower in patients than in control subjects despite normal resting systolic function. ${ }^{6}$ Finally, an additional explanation might be that since most of these patients participated in regular sporting activities, they could be quite fit. However, whatever the cause, the normality of $\mathrm{VO}_{2}$ max and all other variables provides reassurance that no significant functional abnormality exists in these patients. ${ }^{43}$ Therefore, in our opinion these patients should be directed towards physical dynamic activity with no particular limitations. It is important to point out that in our patients we investigated the physical response to dynamic exercise, which primarily causes a volume load on the cardiovascular system, but the response to static exercise, which primarily causes a pressure load on the cardiovascular system, could be quite different.

Table 3 Cardiopulmonary exercise testing parameters in cancer survivors and normal controls by age at test

\begin{tabular}{|c|c|c|c|c|c|c|}
\hline & \multicolumn{2}{|l|}{ Total } & \multicolumn{2}{|l|}{$<13 y$} & \multicolumn{2}{|l|}{$13-21$ y } \\
\hline & Survivors $(n=84$ ) & Controls $(n=79)$ & Survivors $(n=33$ ) & Controls $(n=47)$ & Survivors $(n=51)$ & Controls $(n=32$ ) \\
\hline $\mathrm{VO}_{2} \max \left(\mathrm{ml} / \mathrm{kg} / \mathrm{m}^{2}\right)$ & $36.5(7.7)$ & $38.2(8.2)$ & $34.0(5.7)$ & $38.9(6.7)^{*}$ & $38.1(8.4)$ & $37.1(10)$ \\
\hline VO2 AT $\left(\mathrm{ml} / \mathrm{Kg} / \mathrm{m}^{2}\right)$ & $24.4(5.6)$ & $24.4(6.9)$ & $23.6(3.8)$ & $24.6(5.7)$ & $24.9(6.6)$ & $24.2(8.5)$ \\
\hline $\operatorname{VE}\left(1 / \mathrm{m}^{2}\right)$ & $67.9(23.3)$ & $64.5(21.8)$ & $52.1(12.5)$ & $56.4(19.0)$ & $78.2(23.0)$ & $76.3(20.4)$ \\
\hline RER & $1.1(0.1)$ & $1.1(0.1)$ & $1.1(0.1)$ & $1.1(0.1)$ & $1.1(0.1)$ & $1.1(0.1)$ \\
\hline $\mathrm{O}_{2}$ pulse (ml/beat) & $10.1(4.1)$ & $9.6(3.8)$ & $6.8(1.8)$ & $7.6(2.7)$ & $12.3(3.8)$ & $12.4(3.6)$ \\
\hline Heart rate & $192(13)$ & $189(17)$ & $193(11)$ & $189(16)$ & $191(14)$ & 189 (17) \\
\hline Systolic BP (mm Hg) & $147(23)$ & $148(25)$ & $132(15)$ & $134(15)$ & $157(22)$ & $168(23)$ \\
\hline
\end{tabular}

$\mathrm{VO}_{2}$ max, oxygen consumption at peak exercise; $\mathrm{VO}_{2} \mathrm{AT}$, oxygen consumption at anaerobic threshold; $\mathrm{VE}$, minute ventilation at peak exercise; RER, respiratory exchange ratio at peak exercise; $\mathrm{BP}$, blood pressure at peak exercise. ${ }^{*} \mathrm{p}<0.001$. 


\section{What is already known on this topic}

- Exercise capacity can be reduced, even in apparently healthy survivors of paediatric cancer by several causes

- Such patients are commonly restricted in their physical activities

\section{What this study adds}

- The cardiopulmonary exercise test is useful for the functional evaluation of these patients

- Recommendations regarding the level of participation in physical activities are given, based on the results of the cardiopulmonary exercise test

In the subgroup $<13$ years of age, male patients showed a mean $\mathrm{VO}_{2}$ max slightly but significantly lower than controls. In this subgroup of younger patients, in which the median interval between completion of therapy and test execution was 5.13 years, the mean BMI value was higher than in controls, although this difference did not reach statistical significance. In males the BMI was $>95$ th centile in $11 / 21$ cases; among these were all six patients that performed with $\mathrm{VO}_{2}$ max values $<5$ th centile observed among controls. In females, BMI was $>95$ th centile in $2 / 12$ patients. The majority of our patients were acute lymphoblastic leukaemia survivors. These patients tend to experience increases in weight, relative weight, and body fat mass, especially in the first years after cessation of therapy. ${ }^{211}{ }^{47}$ One of the several contributing factors to this is a decreased physical activity, that in some cases depends on a psychological attitude. ${ }^{3}$ Therefore, considering that all the other exercise parameters were not significantly different from those of controls, the reduction of the aerobic capacity observed in our patients, at least when overweight, was likely to be due to poor physical fitness. Surprisingly, the difference was noted only in males, possibly because of the higher activity level in boys than in girls. ${ }^{39}$ However, we believe that a new CPET performed after an aerobic conditioning programme is highly recommended in such patients, which could be directed towards an unrestricted aerobic activity in case of normal $\mathrm{VO}_{2}$ max, or an activity tailored below their anaerobic threshold. Obviously long term follow up of these patients is warranted.

\section{Authors' affiliations}

E De Caro, A Smeraldi, M Saitta, G Pongiglione, Department of Cardiology, Giannina Gaslini Children's Hospital, Genoa, Italy F Fioredda, G Hanau, M Faraci, F Grisolia, G Dini, Department of Hematology and Oncology, Giannina Gaslini Children's Hospital, Genoa, Italy

M G Calevo, R Haupt, Epidemiology and Biostatistics Section, Scientific Directorate, Giannina Gaslini Children's Hospital, Genoa, Italy

Competing interests: none declared

\section{REFERENCES}

1 Williams CL, Hayman LL, Daniels SR, et al. Cardiovascular health in childhood: a statement for health professionals from the Committee on Atherosclerosis, Hypertension, and Obesity in the Young (AHOY) of the Council on Cardiovascular Disease in the Young, American Heart Association. Circulation 2002;106: 143-60.

2 Talvensaari KK, Knip M, Lanning P, et al. Clinical characteristics and factors affecting growth in long-term survivors of cancer. Med Pediatr Oncol 1996;26:166-72.
3 Calzolari A, Baronci C, Biondi G, et al. Evaluation of a group of leukaemic children "off-therapy", towards their inclusion in physical activities. Int J Sports Cardiol 1985;2:108-15.

4 Pihkala J, Happonen JM, Virtanen $\mathrm{K}$, et al. Cardiopulmonary evaluation of exercise tolerance after chest irradiation and anticancer chemotherapy in children and adolescents. Pediatrics 1995;95:722-6.

5 Larsen R, Barber G, Heise CT, et al. Exercise assessment of cardiac function in children and young adults before and after bone marrow transplantation. Pediatrics 1992;89:722-9.

6 Johnson D, Perrault $\mathrm{H}$, Fournier A, et al. Cardiovascular responses to dynamic submaximal exercise in children previously treated with anthracycline. Am Heart J 1997; 133: 169-73.

7 Sato T, Harada K, Tamura M, et al. Cardiorespiratory exercise capacity and its relation to a new Doppler index in children previously treated with anthracycline. J Am Soc Echocardiogr 2001;14:256-63.

8 Bleyer WA. The impact of childhood cancer on the United States and the world. CA Cancer I Clin 1990;40:355-67.

9 Jenney MM, Faragher B, Morris Jones $\mathrm{PH}$, et al. Lung function and exercise capacity in survivors of childhood leukaemia. Med Pediatr Oncol 1995; 24:222-30

10 Shan K, Lincoff AM, Young JB. Anthracycline induced cardiotoxicity. Ann Intern Med 1996; 125:47-58.

11 Praga C, Beretta G, Vigo PL, et al. Adriamycin cardiotoxicity a survey of 1273 patients. Cancer Treat Rep 1978;63:827-34.

12 Ewer MS, Ali MK, McKay B. A comparison of cardiac biopsy grades and ejection fraction in patients receiving doxorubicin. $J$ Clin Oncol 1984;2:112-17.

13 Lang D, Hilger F, Binswanger J, et al. Late effects of anthracycline therapy in childhood in relation to the function of the heart at rest and under physical stress. Eur J Pediatr 1995;5:340-5.

14 Yeung ST, Yoong C, Spink J, et al. Functional myocardial impairment in children treated with anthracyclines for cancer. Lancet 1991;6:816-18.

15 Washington RL. Cardiopulmonary sequelae after bone marrow transplantation. J Pediatr 2000; 136:280-2.

16 Perry MC. The chemotherapy source book, 3rd edn. Lippincott Williams and Wilkins, 2001:462

17 Cole TJ, Freeman JV, Preece MA. Body mass index references curves for the UK, 1990. Arch Dis Child 1995; 73:25-9.

18 Kremer LCM, van der Pal HJH, Offringa M, et al. Frequency and risk factors of subclinical cardiotoxicity after anthracycline therapy in children; a systematic review. Ann Oncol 2002;13:819-29.

19 Cumming GR, Everatt D, Hastman L. Bruce treadmill test in children; normal values in a clinic population. Am J Cardiol 1978;41:69-75.

20 Steinherz IJ, Steinherz PG, Tan C, et al. Cardiac toxicity 4 to 20 years after completing anthracycline therapy. JAMA 1991;266:1672-7.

21 Lipshultz SE, Colan SD, Gelber RD, et al. Late cardiac effects of doxorubicin therapy for acute lymphoblastic leukemia in childhood. N Engl J Med 1991;324:808-15.

22 Ali MK, Ewer MS, Gibbs HR, et al. Late doxorubicin-associated cardiotoxicity in children. Cancer 1994:74:182-8.

23 Lipshultz SE, Sallan SE. Cardiovascular abnormalities in long-term survivors of childhood malignancy. J Clin Oncol 1993;11:1199-203.

24 Eames GM, Crosson J, Steinberger J, et al. Cardiovascular function in children following bone marrow transplantation: a cross-sectional study. Bone Marrow Transplantation 1997;19:61-6.

25 Weesner KM, Bledsoe M, Chauvenet $A$, et al. Exercise echocardiography in the detection of anthracycline cardiotoxicity. Cancer 1991;68:435-8.

26 De Wolf D, Suys B, Matthys D, et al. Stress echocardiography in the evaluation of late toxicity after moderate dose of anthracycline therapy in childhood. Int J Pediatr Hematol Oncol 1994; 1:399-404.

27 Smibert E, Carlin JB, Vidmar S, et al. Exercise echocardiography reflects cumulative anthracycline exposure during childhood. Pediatr Blood Cancer 2004:42:556-62.

28 Klewer SE, Goldberg SJ, Donnerstein RL, et al. Dobutamine stress echocardiography: a sensitive indicator of diminished myocardial function in symptomatic doxorubicin-treated long-term survivors of childhood cancer. $J$ Am Coll Cardiol 1992; 19:394-401.

29 De Wolf D, Suys B, Verhaaren H, et al. Low-dose dobutamine stress echocardiography in children and young adults. Am J Cardiol 1998;81:895-901.

30 Cottin $Y$, L'Hulilier I, Casasnovas $O$, et al. Dobutamine stress echocardiography identifies anthracycline cardiotoxicity. Eur J Echocardiography 2000;1:180-3.

31 Harrington D, Anker SD, Coats JS. Preservation of exercise capacity and lack of peripheral changes in asymptomatic patients with severe impaired left ventricular function. Eur Heart $J$ 2001; 22:392-9.

32 Fang $Z Y$, Marwick TH. Mechanisms of exercise training in patients with heart failure. Am Heart J 2003;145:904-11.

33 Lipshultz SE, Colan SD. Cardiovascular trials in long-term survivors of childhood cancer. I Clin Oncol 2004;22:769-73.

34 Wassermann K, Hansen JE, Sue DY, et al. Chapter 7. Principles of exercise testing and interpretation, 3rd edn. Lippincott Williams and Wilkins, 1999.

35 Weisman IM, Zeballos RJ. An integrated approach to the interpretation of cardiopulmonary exercise testing. Clinics in Chest Medicine 1994; 15:421-45.

36 Hauser M, Gibson BS, Wilson N. Diagnosis of anthracycline-induced late cardiomyopathy by exercise-spiroergometry and stress-echocardiography. Eur J Pediatr 2001;160:607-10.

37 Jenney MM, Faragher $\mathrm{B}$, Morris Jones $\mathrm{PH}$, et al. Lung function and exercise capacity in survivors of childhood leukaemia. Med Pediatr Oncol $1995 ; 24: 222-30$ 
38 Hovi L, Er P, Rautonen J, et al. Impaired muscle strength in female adolescents and young adults surviving leukemia in childhood. Cancer 1992;72:276-81.

39 Matthys D, Verhaaren H, Benoit Y, et al. Gender difference in aerobic capacity in adolescents after cure from malignant disease in childhood. Acta Paediatr 1993:82:459-62

40 Black P, Gutjahr P, Stopfkuchen H. Physical performance in long-term survivors of acute leukaemia in childhood. Eur J Pediatr 1998;157:464-7.

41 Zee $\mathbf{P}$, Chen $\mathrm{CH}$. Prevalence of obesity in children after therapy for acute lymphoblastic leukemia. Am J Pediatr Hematol Oncol 1986:8:294-9.

42 Ettinger RS, Heiney SP. Cancer in adolescents and young adults. Psychosocial concerns, coping strategies, and interactions. Cancer 1993;71:3276-80.
43 Weisman IM, Zeballos RJ. Cardiopulmonary exercise testing. Pulmonary and Critical Care Update PCCU 1995;11:2.

44 Sharkey AM, Carely $A B$, Heise $C T$, et al. Cardiac rehabilitation after cancer in children and young adults. Am J Cardiol 1993:71:1488-90.

45 Silber JH, Jakacki R, Larsen $\mathrm{PL}$, et al. Increased risk of cardiac dysfunction after anthracyclines in girls. Med Pediatr Oncol 1993;21:477-9.

46 Steinherz $\mathrm{L}$. Anthracycline-induced cardiotoxicity. Ann Intern Med 1997: 126:827-8

47 Turner-Gomes SO, Lands LC, Halton J, et al. Cardiorespiratory status after treatment for acute lymphoblastic leukemia. Med Pediatr Oncol $1996 ; 26: 160-5$.

\section{IMAGES IN PAEDIATRICS}

\section{Smuggling contraband drugs using paediatric "body packers"}

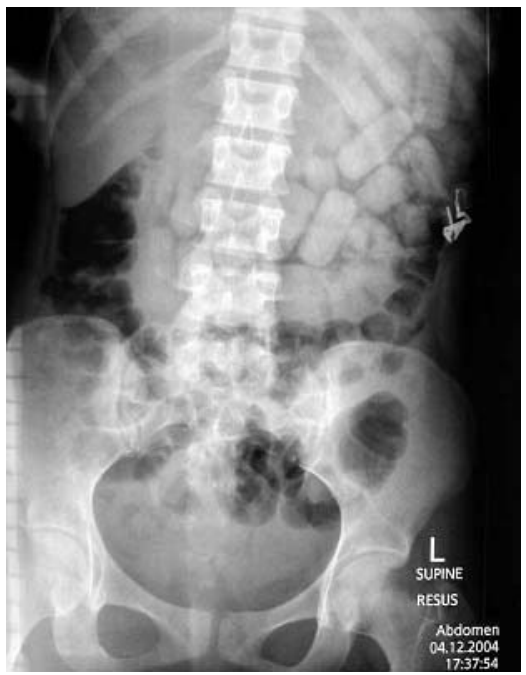

Figure 1 The intra-abdominal radio-opacities suggestive of "body-packing". teenage girl was brought to the accident and emergency unit by airport officials after being found unconscious in her seat on a flight landing at Heathrow from Nigeria. On presentation her sensorium on the Glasgow Coma Scale was 6/15. She was hypothermic (temperature $35.3^{\circ} \mathrm{C}$ ) and her pupils were constricted bilaterally.

An $x$ ray examination of the abdomen revealed multiple cylindrical opacities as seen with body packing (fig 1). Body packing is a term used to signify the smuggling of illicit drugs using the human body as a vehicle. Individual packets of drugs (usually cocaine or heroine) are wrapped in waterproof material such as latex glove fingers or condoms, and swallowed.'

Intravenous naloxone $(400 \mu \mathrm{g})$ was administered and her sensorium improved immediately. However she lapsed back into unconsciousness soon afterwards. A naloxone infusion was started (running at $1000 \mu \mathrm{g} /$ hour) and she was transferred to a tertiary unit where whole bowel irrigation with polythene glycol was administered until all the bags were expelled.

Body packing has been used as an important means of international drug trafficking in several large cities all over the world, but it is extremely rare in the paediatric age group. There are only two cases reported in children under $16 .{ }^{1}$ Drug toxicity owing to leaking or rupture of the packets, and intestinal obstruction have been reported. ${ }^{2}$ The $x$ ray picture is usually diagnostic with a sensitivity of up to $90 \%{ }^{2}$ Paediatricians also now need to be aware of this radiological appearance.

A Chakrabarty, S Hydros, J M Puliyel West Middlesex University Hospital, London, UK; puliyel@vsnl.com

Competing interests: none declared

\section{REFERENCES}

1 Traub SJ, Kohn GL, Hoffman RS, et al. Pediatric "body packing". Arch Pediatr Adolesc Med 2003;157:174-7.

2 Traub SJ, Hoffmann RS, Nelson LS. Body packing -the internal concealment of illicit drugs. N Engl J Med 2003;349:2519-26. 